\title{
KLYPVE-EUSO: Science and UHECR observational capabilities
}

\author{
M. Casolino*1, A. Belov' ${ }^{2}$, M. Bertaina ${ }^{5}$, T. Ebisuzaki ${ }^{6}$, M. Fukushima ${ }^{4}$, P. Klimov², M. \\ Panasyuk $^{2}$, P. Picozza ${ }^{3}$, H. Sagawa ${ }^{4}$ K. Shinozaki ${ }^{5}$, for the JEM-EUSO collaboration \\ ${ }^{1}$ INFN and RIKEN, ${ }^{2}$ Lomonosov Moscow State University, Skobeltsyn Institute of Nuclear \\ Physics (SINP MSU), Moscow, Russia, ${ }^{3}$ University of Rome Tor Vergata, Rome, ${ }^{4}$ ICRR, Tokyo \\ University, Japan, ${ }^{5}$ INFN and University of Torino, Italy, ${ }^{6}$ Riken, Japan, ${ }^{7}$ INFN LNF, Italy \\ E-mail: casolino@roma2.infn.it
}

\begin{abstract}
The study of Ultra High Energy Cosmic Rays (UHECRs) offers unique possibilities to probe the energies currently inaccessible by man-made accelerators. The last years have shed light on several characteristics of these particles, but - due to their extremely low flux - their origin, nature and acceleration mechanisms are still unclear. K-EUSO will be the detector to perform measurement of the flux of UHECR from space, covering and comparing the spectra of both Northern and Southern Hemispheres. In this work we will discuss the science capabilities of the detector.
\end{abstract}

35th International Cosmic Ray Conference - ICRC2017

10-20 July, 2017

Bexco, Busan, Korea

${ }^{*}$ Speaker. 


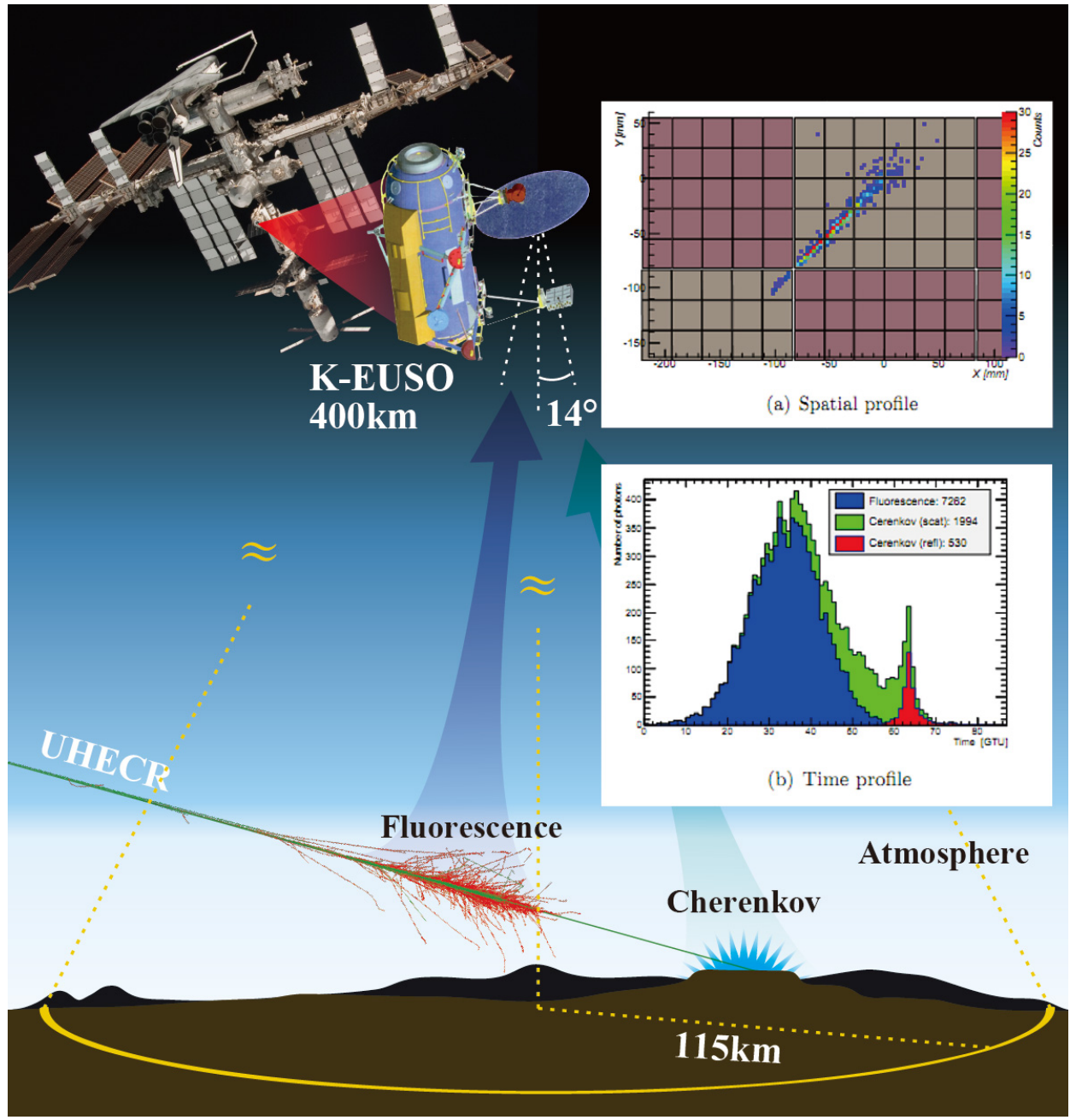

Figure 1: The concept of the K-EUSO detector to observe an UHECR from space. The UV light emitted in an extensive air shower is detected by K-EUSO. From the temporal and spatial profile of the shower the energy and arrival direction of the primary particle is reconstructed. Upper Right: The time and spacial resolution of an extensive airshower with the energy of $10^{20} \mathrm{eV}$ and the azimuthal angle of $60^{\circ}$. A square represents a MAPMT with 64 channels, arranged in units of $6 \times 6$ phototubes to form photodetector module (PDM). The small squares show the number of photons detected by each channel of the MAPMT. This event starts from the lower-left corner continues to the right top of the PDM. Lower Right: Time profile of photons, obtained in GTUs $(2.5 \mu \mathrm{s})$. The signal consists of three components (UV light -blue, Cherenkov peak - green, scattered Cherenkov - red).

\section{Introduction}

K-EUSO continues the Russian program for Ultra High Energy Cosmic Rays (UHECRs) studies, begun with the UV detectors installed on board Tatiana-1, Tatiana-2 and Vernov satellites. These were followed by TUS[5], the first attempt to measure UHECR fluorescent light from space (Figure 1). 
The fundamental objectives of K-EUSO are the demonstration of the space-based observation of UHECRs and the study of the anisotropy in arrival direction of UHECRs across the whole celestial sphere.

Furthermore, K-EUSO will investigate phenomena intrinsic to the Earth's atmosphere or induced by the meteoroids incoming from space as well as search for strange quark matter.

K-EUSO is a result of the joint efforts to improve the performance of the Russian KLYPVE mission, by employing the technologies developed for the JEM-EUSO mission, such as the focal surface detectors and the readout electronics. The KLYPVE mission, named after Russian word "ultra-high energy cosmic rays," was selected by the Space Program by the Russian Federation and has undergone pre-phase A study, including launch and accommodation on the ISS. Since its first conception as KLYPVE, K-EUSO project has passed various modifications aimed to increase FOV and UHECR statistics [7, 8].

It will be the first detector with a real capability for UHECR spectrum and anisotropy study with a sufficient statistics and the full celestial sphere coverage.

The adopted optical layout is a Schmidt camera covering a field of view of $40^{\circ}$ with an entrance pupil diameter of $2.5 \mathrm{~m}$, a $4 \mathrm{~m}$ diameter spherical mirror and a focal length of $1.7 \mathrm{~m}$ (see Figure 2)[8].

The temporal and spatial evolution of UV light recorded by K-EUSO will allow the reconstruction of the EAS, allowing the energy and arrival direction of the UHECR to be determined. The camera focal plane is covered by $1.2 \times 10^{5}$ pixels, each smaller than $2.88 \times 2.88 \mathrm{~mm}$, giving a $0.066^{\circ}$ angular resolution per pixel; a pixel covers about $0.8 \mathrm{~km}$ on the surface of the Earth for ISS altitude of $400 \mathrm{~km}$. Sampling time is $2.5 \mu$ s (Figure 1).

Attached to the Russian MRM-1 module on-board ISS (Figure 2), it will detect UHECRs above $2 \times 10^{19} \mathrm{eV}$ with a yearly exposure of about 4 times Auger (Figure 3), with an exposure flat over the whole sky (Figure 4). K-EUSO is planned to operate for minimum of 2 years and it can function more than 6 years if the lifetime of the ISS is extended[6].
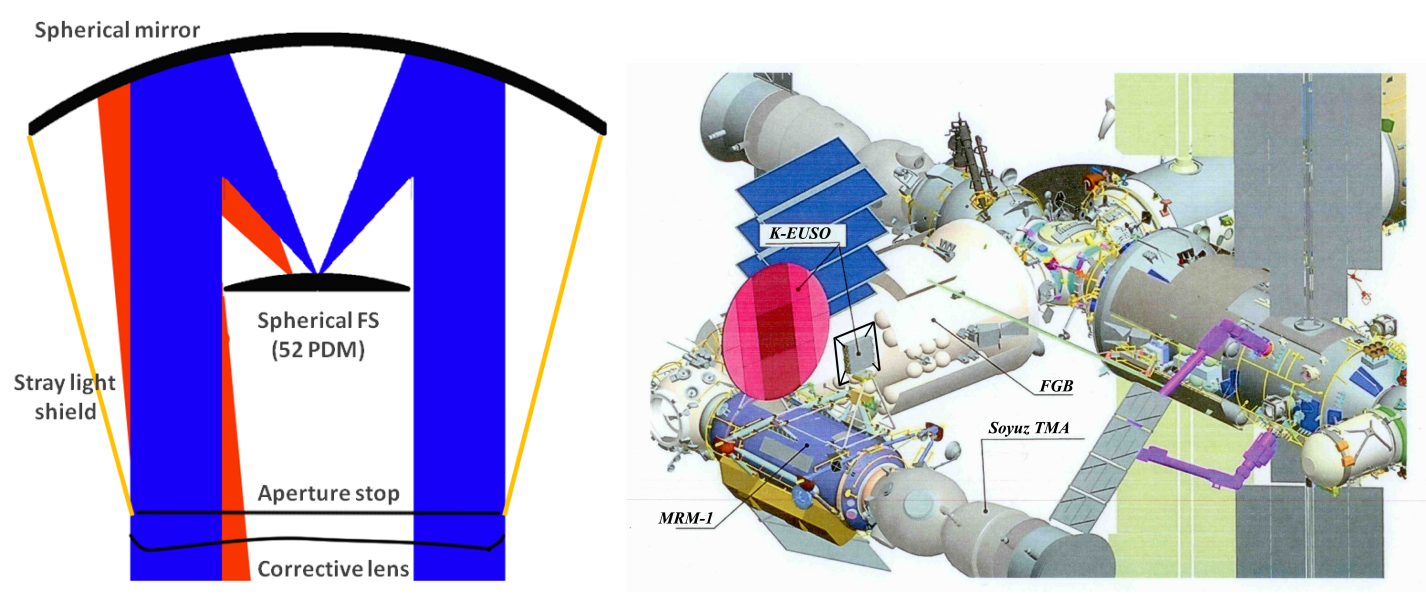

Figure 2: Left: Scheme of K-EUSO Schmidt optics, with the spherical reflector (4m diameter) on top and corrective lens and spherical focal surface on the bottom. Right: Location of K-EUSO on the MRM-1 module of the Russian section of the ISS. 


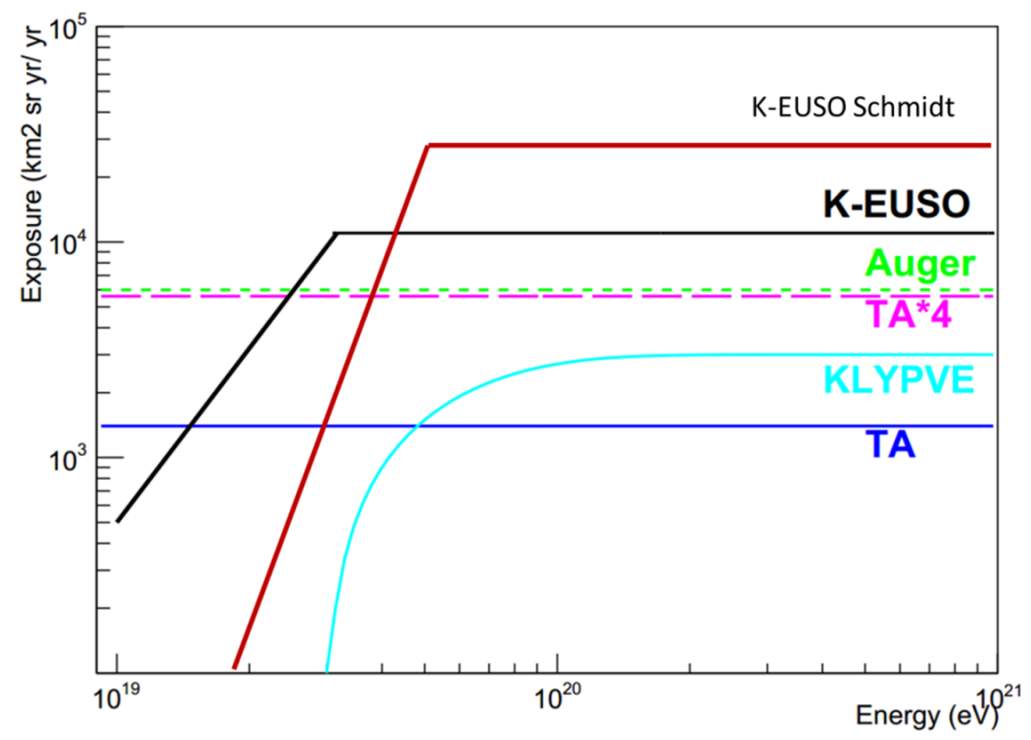

Figure 3: Comparison of the exposures of the various ground based and space-borne detectors. K-EUSO with Schmidt optics offers a higher exposure at higher energies with a higher minimum energy threshold of particles detected due to the larger shadow of the Focal Surface.

\section{Science objectives}

The main goals of K-EUSO for UHECR observations are:

1. To perform the first all-sky observation of the UHECR, in order to establish whether - and from what energy - the particle fluxes of the two hemispheres are different.

Northern and southern skies are very asymmetric at the highest energy region: assuming TA and Auger spectra, the expected number of K-EUSO events per year $\left(\mathrm{E}>5.7 \times 10^{19} \mathrm{eV}\right)$ is 143 in the northern hemisphere and 30 events in the southern hemisphere, whereas almost the same number of events would be expected in both hemispheres from the isotropic sky. The difference in flux arises partially from the overall energy scale difference between TA and PAO ${ }^{1}$ so - if the difference in spectra is only due to energy scaling - the expected number of events could range from 60 to 246 per year over the whole sky. In case of a different flux. Even after adjusting the relative energy scale, however, the fluxes above $10^{19.5} \mathrm{eV}$ significantly differ in both ground experiments. Whether the remaining difference, a large N-S dipole anisotropy, is due to the intrinsic physics or the measurement systematics is one of very important questions the present ground experiments are facing. The measurement of K-EUSO will give a definite answer to this question after the first six months of operation.

An all-sky measurement will have a low systematics in the comparison of the particle fluxes in the two hemispheres. K-EUSO will then have to be cross-calibrated with the ground arrays, normalizing the absolute flux at low energy, (at $\simeq 3 \times 10^{19} \mathrm{eV}$ to study differences in

\footnotetext{
${ }^{1}$ The spectra of TA and Auger in $10^{18.2} \mathrm{eV}-10^{19.5} \mathrm{eV}$ coincide well by shifting the energy scale of either experiment by approximately $8.5 \%$. [9]
} 


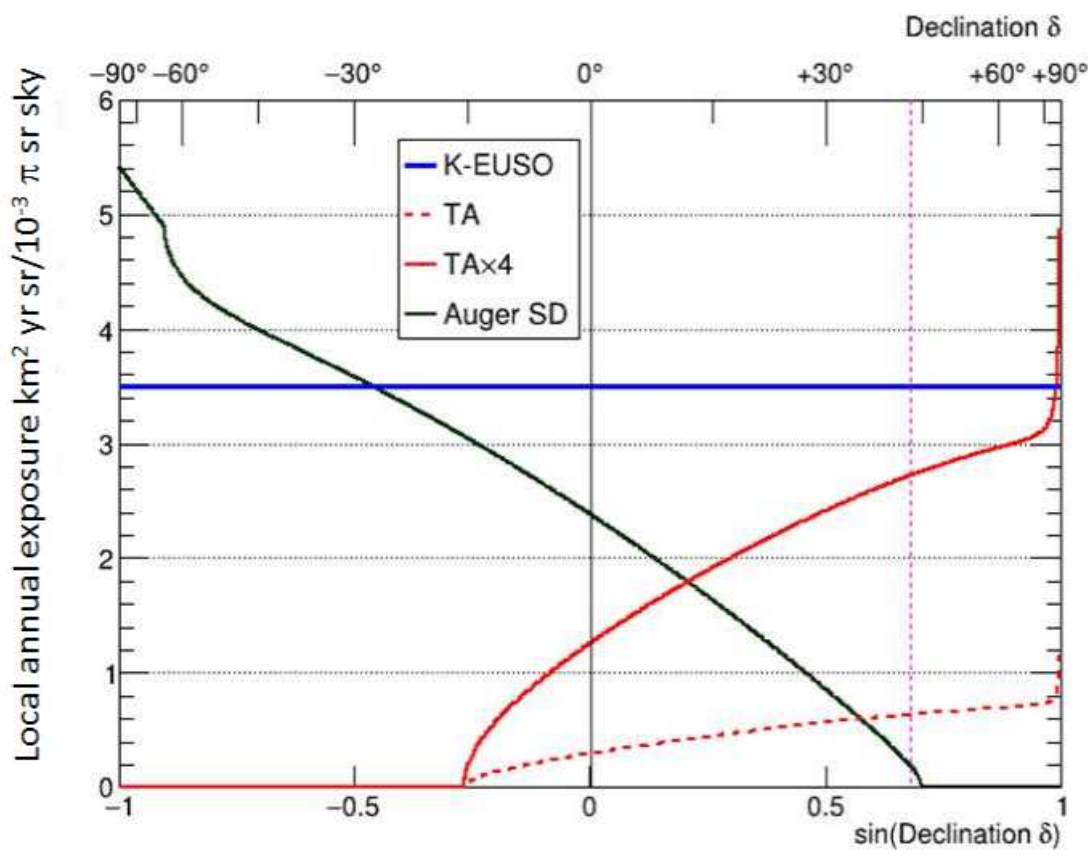

Figure 4: Annual exposure $\left(\mathrm{km}^{2} \mathrm{yr} \mathrm{sr}\right)$ in bins of $10^{-3}$ radian for the declination angle $(\delta)$ vs. $\sin (\delta)$ for K-EUSO, TA, TA $\times 4$ and Auger. One can see the flatter response of K-EUSO compared with the one of the two ground detectors.

the spectral shape among the two hemispheres, especially at the highest energy region). See Figure 5 for the number of events expected in each hemisphere after one year of observation.

2. To analyse UHECR clustering to independently confirm (or rule out) the presence of hotspots in the northern and southern hemispheres, and compare their spectral indexes.

K-EUSO's second physics target is independently confirming, or refuting, TA hotspot at $(\alpha, \delta)=\left(146.7^{\circ}, 43.2^{\circ}\right)$, and PAO warm spot in the Cen A direction. After one-year of observation TA hotspot will be confirmed at the $3 \sigma$ level.

3. To study possible UHECR anisotropies over various angular scales, including possible correlation to nearby large-scale extragalactic structures ( $\approx 40$ degrees), Milky-Way related features, and dipole-type anisotropy.

The key of resolving the origin of UHECRs and understanding the mechanisms of acceleration and propagation in extragalactic space lies in the study of their angular distribution and the anisotropy on the celestial sphere. If we are dealing with astrophysical sources, the closest objects should dominate the scene or at least contribute to some anisotropy in the sky. If the sky is mostly isotropic this would point in favor of some kind of cosmological model involving ultra-heavy dark matter and top down production of UHECR (see, for instance [2]).

Given the anisotropy of the nearby ( $\simeq 100 \mathrm{Mpc}$ ) universe, diffusive propagation of UHECRs from nearby extragalactic sources would probably result in a non-zero dipole flux. Fur- 


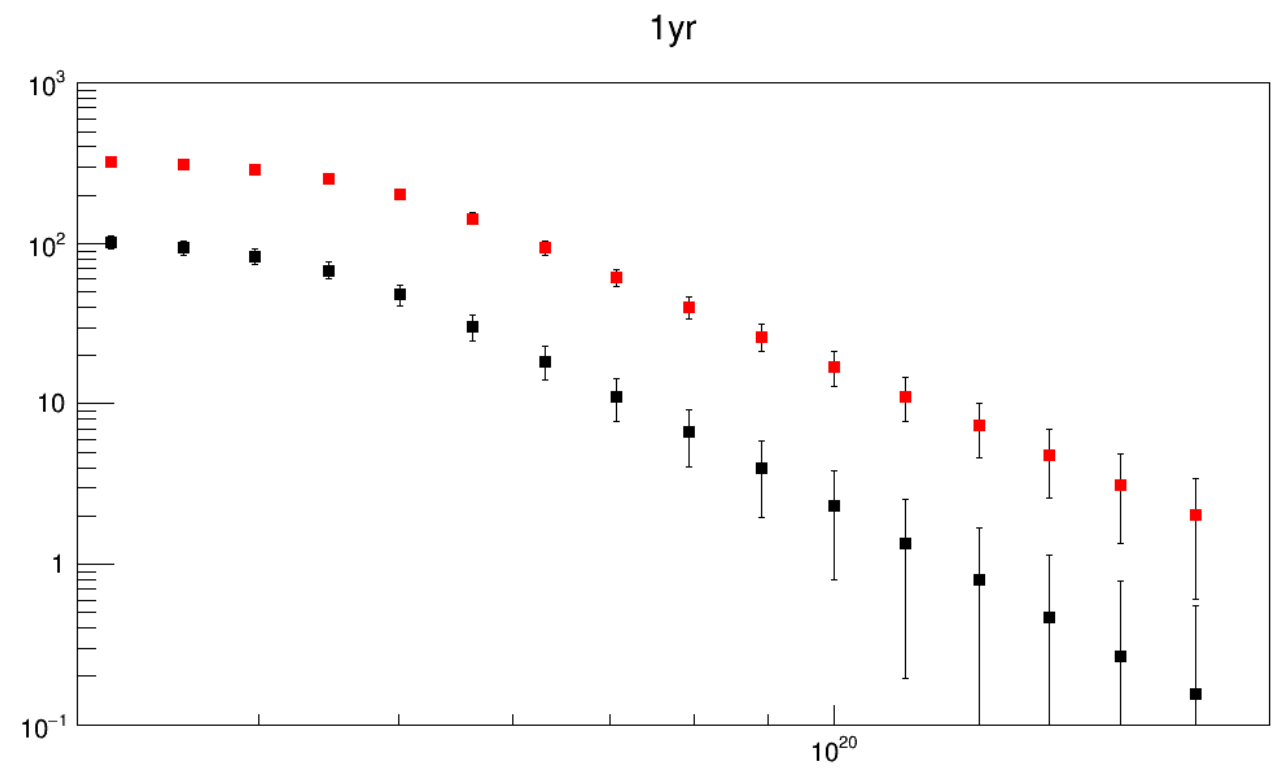

Figure 5: Integral number spectra (number of events $\mathrm{N}$ above the energy $\mathrm{E}(\mathrm{eV})$ ) as observed on each Hemisphere of the whole sky after one year of K-EUSO observation. The red points represent number of events in the North assuming TA spectrum in ICRC2015, the black points corresponds to Southern Hemisphere events using Auger spectrum (same conference). Statistical error bars are shown.

thermore, excesses along a plane, e.g. the super-Galactic one, would be detectable as a quadrupole structure. The result of joint TA-PAO study for the dipole anisotropy [1] above 1 $\times 10^{19} \mathrm{eV}$ can be confirmed by K-EUSO with much reduced systematics. K-EUSO opens a possibility of anisotropy to study at the highest energy region, above $5.7 \times 10^{19} \mathrm{eV}$, and also by combining and unifying the measurements of all experiments to obtain improved statistics (Figure 6). Particles of higher energy are less bent by magnetic fields, and the anisotropy should probably grow with energy, making the measurement at $\mathrm{E}>5.7 \times 10^{19} \mathrm{eV}$ all the more relevant.

Quadrupole and higher order spherical harmonic structures in the sky can also be addressed by K-EUSO: we could detect the presence of these structures within a few years from launch. A null result for large scale anisotropy in the high energy region, on the other hand, would point in favour of cosmological top-down origin of UHECRs[2].

These objectives will be reached after six months of data gathering in flight and significance will be improved after two years of flight[4]. In case of prolongation of the flight of the ISS to 2028, a much larger statistics will be gathered.

Other science objectives involve:

1. Search for Strange Quark Matter and study of Meteoroids

2. Search and observation of Space Debris

3. Study of UV Emission of Night-Time Earth 
4. Study of fast component of Transient Luminous Events

For a detailed discussion of these objectives see [3].

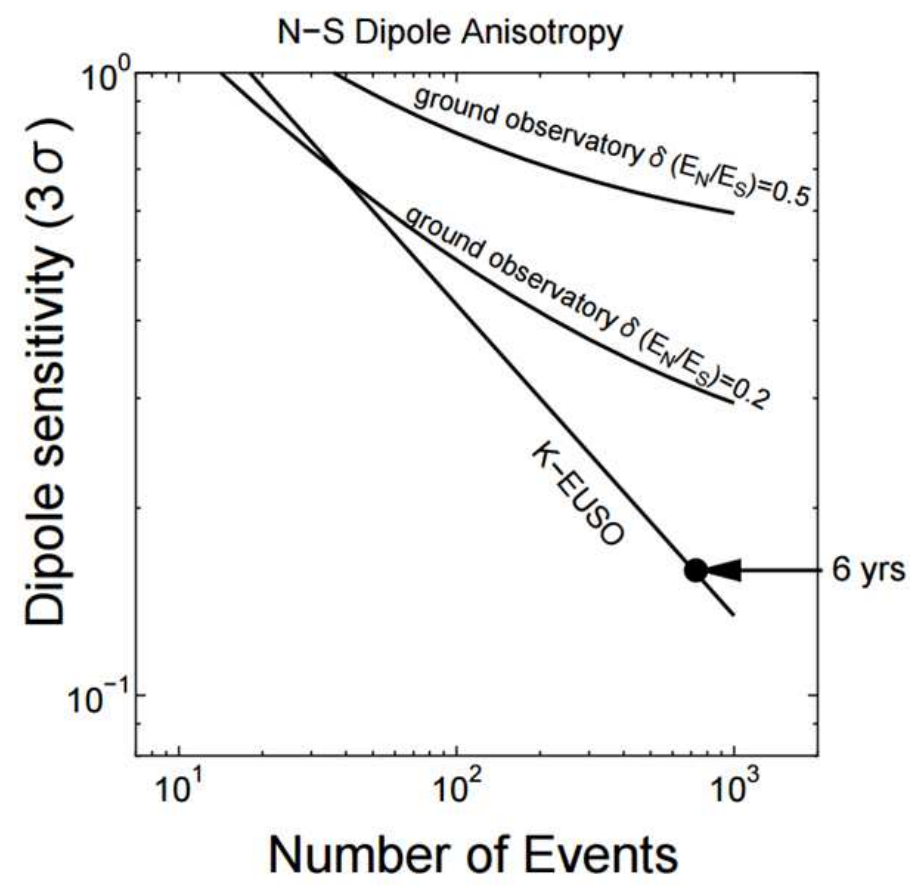

Figure 6: Dipole sensitivity ( $3 \sigma$ level) of KLYPVE-EUSO compared with the ground observatories above $5.7 \times 10^{19} \mathrm{eV}$. By 6 years of data (496 events in total), it will reach well below $20 \%$. Ground observatories have larger systematic errors caused by those of the effective exposure and the energy calibration between north and south observatories. The two curves of the ground observatories refer to $50 \%$ (top) and $20 \%$ (bottom) error in an effective exposure ratio, which includes energy scale uncertainty.

\section{Acknowledgments}

This work was partially supported by Basic Science Interdisciplinary Research Projects of RIKEN and JSPS KAKENHI Grant (22340063, 23340081, and 24244042), by the Italian Ministry of Foreign Affairs and International Cooperation, by the Italian Space Agency through the ASI INFN agreement $n$. 2017-8-H.0, by contract contract 2016-1-U.0, by NASA award 11-APRA-0058 in the USA, by the Deutsches Zentrum fÂlur Luft- und Raumfahrt, by the French space agency CNES, the Helmholtz Alliance for Astroparticle Physics funded by the Initiative and Networking Fund of the Helmholtz Association (Germany), and by Slovak Academy of Sciences MVTS JEMEUSO as well as VEGA grant agency project 2/0132/17. Russia is supported by ROSCOSMOS and the Russian Foundation for Basic Research Grant No 16-29-13065, and the Olle Engkvist Byggmästare Foundation.

We dedicate this proceeding to Yoshiya Kawasaki and Jacek Karczmarczyk, who passed away in 2016. 


\section{References}

[1] Abbasi, R. U., et al. Pierre Auger Observatory and Telescope Array: Joint Contributions to the 34th International Cosmic Ray Conference (ICRC 2015). ArXiv e-prints 1511.02103, November 2015.

[2] Addazi, A. et al. Neutron Majorana mass from exotic instantons. Journal of High Energy Physics, 12:89, December 2014. doi:10.1007/JHEP12(2014)089.

[3] Casolino, M. Science of Mini-EUSO detector on board the International Space Station. These proceedings.

[4] Ebisuzaki, T. K-EUSO proposal. 2014.

[5] Klimov, P. Results of the TUS detector. These proceedings.

[6] Klimov, P. Status of the KLYPVE-EUSO detector for UHECR study on board the ISS. These proceedings.

[7] Panasyuk, M., et al. The current status of orbital experiments for UHECR studies. Journal of Physics: Conference Series, 632(1), 2015. doi:10.1088/1742-6596/632/1/012097.

[8] Sandri, P. K-EUSO Schmidt type optical system for the KLYPVE-EUSO UHECR detector. These proceedings.

[9] Valino, I. The flux of ultra-high energy cosmic rays after ten years of operation of the Pierre Auger Observatory. proceedings of the 34th ICRC, Den Haag (2015). 